\title{
Aquaporin, forward osmosis and biomimetic membranes
}

\author{
Nikolai Kocherginsky \\ Biomime, Urbana, Illinois, USA
}

\begin{abstract}
Aquaporin attracted attention not only of physiologists and biophysicists, but also of chemical engineers. Here we critically analyze a paper describing aquaporin-based artificial membranes, suggested for forward osmosis-based water purification (Wang et al. 2012, Small 8, pp. 1185-1190). Related papers published later by the same group are also discussed. We indicate recently developed general approach to describe membrane transport, membrane permeability and selectivity, which is applicable for forward osmosis. In addition, we also mention our papers describing simple nitrocellulose-based membranes, which have selective aqueous channels without proteins, but successfully imitate many properties of biomembranes.
\end{abstract}

Key words: Biomimetic membrane - Aquaporin - Water transport - Forward osmosis - Nonequilibrium thermodynamics

\section{Introduction}

Osmosis and transmembrane water transport play an immense role in physiology and biophysics, and one of the great breakthroughs in this area of research was the 1991 discovery by P. Agre of the long-sought aqueous "channels", proteins that regulate and facilitate water transport through cell membranes. These proteins are called aquaporins and the work was awarded the 2003 Nobel Prize in Chemistry (Agre 2004).

The very first Nobel Prize in Chemistry (1901) was given for the brilliant work on osmotic pressure and chemical equilibriums to Jacobus Henricus van't Hoff. Modern English translation of his paper, published in 1887, may be found in a special $100^{\text {th }}$ volume of Journal of Membrane Science (van't Hoff 1995). Later it became clear that it is also possible to apply an external pressure difference through the membrane and remove water from brine in reverse osmosis (RO). Applied hydrostatic pressure difference in $\mathrm{RO}$ must exceed the osmotic pressure difference and have an opposite sign. Fifty years ago Sydney Loeb together with Srinivasan Sourirajan described the first asymmetric cellulose acetate membrane where a thin and dense water-selective layer was formed on a much more porous mechanical support (Loeb and Sourirajan 1963). The polymer membrane had high permeability

Correspondence to: Nikolai Kocherginsky, 909 E. Sunnycrest Drive, Urbana, Illinois 61801, USA

E-mail: nm_koch@yahoo.com for water and high water/salt selectivity, which made reverse osmosis a practical technology. World-wide production of $\mathrm{RO}$ water now is more than 6.5 million $\mathrm{m}^{3} /$ day.

It seems like an attractive idea to form a thin layer with aquaporin on a porous support and to use it as a membrane for water purification. Recent results published in this area are discussed below.

\section{Results and Discussion}

\section{Aquaporin-based membranes}

Potable water production is especially important for Singapore, and several recent papers from two leading Singapore Universities describe their attempts to reach this goal (Duong et al. 2012; Guofei et al. 2012; Li et al. 2012; Phuoc 2012; Wang et al. 2012a, 2012b, 2013a, 2013b; Xie et al. 2013; Zhao et al. 2012; Zhong et al. 2012; Ge et al. 2013). One of the first papers described the membrane where lipid vesicles with aquaporin were fused on a surface of a commercial hydrophilic nanofiltration membrane (Li et al. 2012). The fusion was driven by hydrostatic pressure and could be facilitated by positively charged lipids adsorbed on the polymer surface. The authors used dead-end filtration with applied pressure difference 1 bar. Though the membranes were interesting as a model system, both pressure-driven water flux and salt rejection only decreased in comparison to the nanofiltration membrane without aquaporin. Nevertheless, $\mathrm{NaCl}$ rejection 
using chemically immobilized proteoliposomes could be as high as 97\% (Zhao et al. 2012).

Professor Chung's group from the National University of Singapore reported that when aquaporin was embedded into a modified cellulose acetate nanofiltration membrane using previously described copolymer vesicles, water permeability in the pressure-driven process decreased at least by an order of magnitude, and the $\mathrm{NaCl}$ rejection was not more than 30\% (Zhong et al. 2012). Similar tendency was observed with porous alumina support (Phuoc et al. 2012). A separate paper from the same group described aquaporin in supported Langmuir monolayers, but it did not characterize water transport (Guofei et al. 2012).

In the next paper the authors claimed that they were able to get a very encouraging water flux using aquaporin-based membranes. New experimental results were presented as a breakthrough and were published in a journal Small, which has a high citation index. The paper has the title "Highly permeable and selective pore-spanning biomimetic membrane embedded with aquaporin Z" (Wang et al. 2012a). Below we will discuss these results together with some other related publications from the same group, mainly published in the Journal of Membrane Science.

Highly permeable triblock polymeric vesicles with membrane-incorporated aquaporin $\mathrm{Z}$ were described earlier by another group (Kumar et al. 2007). To study forward osmosis, the protein was again incorporated into hydrated triblock copolymer vesicles, and then the vesicles were immobilized in the pores of a polycarbonate track etched substrate. The surface of the substrate was additionally coated with $60 \mathrm{~nm}$ thick gold layer with acrylate groups on it, used to chemically immobilize the copolymer vesicles. When the pore size was $50 \mathrm{~nm}$, the average pore-spanning vesicle layer in the membrane was 6-8 $\mathrm{nm}$ (one vesicle per pore), though the initial vesicle diameter before immobilization was near $150 \mathrm{~nm}$. It means that the vesicles were broken and were immobilized at the top of the filter.

The membrane this time was characterized without any external transmembrane pressure, based only on osmosisdriven changes of the solution weight (not volume) with time (Wang et al. 2012a). When the membrane separated pure water and $2 \mathrm{M} \mathrm{NaCl}$ draw solution, water flux $J_{w}$ was as high as $1421 / \mathrm{m}^{2} / \mathrm{h}$. Salt flux in the opposite direction was also perfect (below $10 \mathrm{~g} / \mathrm{m}^{2} / \mathrm{h}$ ), but for some reasons it did not change systematically with salt concentration. If we recalculate water and $\mathrm{NaCl}$ fluxes and present them in the same units, they were $7.9 \times 10^{3} \mathrm{~mol} / \mathrm{m}^{2} / \mathrm{h}$, and $0.17 \mathrm{~mol} / \mathrm{m}^{2} / \mathrm{h}$, giving a very high selectivity $\alpha_{\mathrm{w} / \mathrm{s}}=4.65$ $\times 10^{4}$. With $0.3 \mathrm{M}$ sucrose as a draw solution water flux decreased by $\sim 10$ times. When low concentration of salt was added into water and moved into the draw solution, reported water/salt selectivity (near 7500, Table 1) was 6 times lower than calculated above.
The authors did not give the polycarbonate filter thickness $l_{2}$ and the brand name, but usually commercial filters have thickness above $10 \mu \mathrm{m}\left(10^{-5} \mathrm{~m}\right)$ to be mechanically stable (Baker 2004). Porosity $\rho$ (15\%) was given in the "Supporting Information". Osmotic permeability of the porous support may be estimated using diffusion coefficient in bulk water $\left(\mathrm{D}_{\mathrm{w}} \sim 1.5 \times 10^{-9} \mathrm{~m}^{2} / \mathrm{s}\right)$ :

$$
P_{w}=\frac{D_{w} \rho}{l_{2}} \sim 2.25 \times 10^{-5} \mathrm{~m} / \mathrm{s}
$$

Concentration polarization without external pressure should not play an important role, but anyway it should only decrease the flux. If osmolarity $C$ were $4 \mathrm{osmol} / \mathrm{l}$ in one side of the porous support and zero in another, the water flux should be:

$$
\begin{aligned}
& J_{w}=P_{w} \Delta C=\left(2.25 \times 10^{-5} \mathrm{~m} / \mathrm{s}\right) \times(4 \mathrm{osmol} / \mathrm{l}) \times \\
& \times\left(18 \times 10^{-3} \mathrm{l} / \mathrm{mol}\right)=1.62 \times 10^{-6} \mathrm{~m} / \mathrm{s}
\end{aligned}
$$

Using that $1 \mathrm{~m} / \mathrm{s}=3.6 \times 10^{6} \mathrm{l} / \mathrm{m}^{2} / \mathrm{h}$, it gives $J_{\mathrm{w}} \approx 61 / \mathrm{m}^{2} / \mathrm{h}$. Real mass transfer resistance is total of the resistances for the thin selective layer, the support and unstirred stagnant aqueous layers near the membrane, so that water permeability has to be only less than our estimate. It means that the fluxes reported in journal Small are at least 35 times larger than the maximum theoretical value based on diffusion.

Publication in Small further compared the fluxes through two types of membranes. One was suspension of vesicles, and another was a porous polymer support with vesicles. Using light scattering, the authors determined osmotic water permeability of polymer vesicles with aquaporin, which was near $2 \times 10^{-3} \mathrm{~m} / \mathrm{s}$ (Fig. 1c in Wang et al. 2012a), i.e. much better than with oocytes (see below). Surprisingly, it almost did not change when aquaporin/ polymer ratio changed by four times (See Wang et al. 2012a, "Supporting Information"). For $1 \mathrm{~mol} / \mathrm{l} \mathrm{NaCl}$ it is possible to expect that:

$$
\begin{aligned}
& J_{W}=2 \times 10^{-3} \mathrm{~m} / \mathrm{s} \times\left(2 \times 10^{3} \mathrm{osmol} / \mathrm{m}^{3}\right) \times \\
& \times\left(18 \times 10^{-3} \mathrm{l} / \mathrm{mol}\right) \times 3.6 \times 10^{3} \mathrm{~s} / \mathrm{h}=259 \mathrm{l} / \mathrm{m}^{2} / \mathrm{h}
\end{aligned}
$$

Instead, the authors claimed that the theoretical flux is near $500-800 \mathrm{l} / \mathrm{m}^{2} / \mathrm{h}$ for different vesicles, and that on a supported membrane it was 10-20 times less (Table S4). The problem with this comparison is that in the first case there is water inside the vesicles, which is certainly unstirred, and in the second case there are unstirred water layers in the polymer porous support and near it. In the first case water permeates through the membrane channels into the unstirred layers inside the vesicles, increasing their volume and changing the light scattering, determined by their diameter $(\sim 150 \mathrm{~nm})$. In the supported membrane experiments situation is different and water penetrates 
through the unstirred layers. Usually unstirred aqueous layers have the thickness $10-100 \mu \mathrm{m}$ and certainly should represent an essential additional resistance (Baker 2004). Thus, the true permeability of the supported membranes calculated per unit surface area of aqueous channels and unstirred layers must be much less than reported $1 / 20$ of the value for vesicles.

\section{Role of unstirred layers}

The surface area of aquaporin aqueous channels, taking only small part of the membrane, and that of unstirred water layers in the porous support (Eq. 4, subscripts 1 and 2) are very different. For water transport through two layers, characterized by different area $\left(s_{1}\right.$ and $\left.s_{2}\right)$ and thickness $\left(l_{1}\right.$ and $\left.l_{2}\right)$, using local distribution coefficients $\left(K_{w, 1}, K_{w, 2}\right)$ and diffusion coefficients $\left(D_{w, 1}, D_{w, 2}\right)$, just like for two electric resistances in series, we have:

$$
\frac{1}{P_{w}}=\frac{l_{1}}{s_{1} K_{w, 1} D_{w, 1}}+\frac{l_{2}}{s_{2} K_{w, 2} D_{w, 2}}>\frac{l_{2}}{s_{2} K_{w, 2} D_{w}} \sim \frac{l_{2}}{s_{2} D_{w}}(4)
$$

Here we assume that for aqueous pores in the support $K_{w, 2}=1$ and the diffusion coefficient is the same as in the bulk water. This analysis shows that at high enough content of aquaporin water permeability must be determined not by the thin selective layer with aquaporin but by the underlying porous polymer layer with unstirred water, like it was in Eq. 1.

Permeability of salt (subscript ${ }_{s}$ ) is described by similar equation, but it is limited by thin selective layer with aquaporin, because it has very low salt permeability:

$$
\frac{1}{P_{s}}=\frac{l_{1}}{s_{1} K_{s, 1} D_{s, 1}}+\frac{l_{2}}{s_{2} K_{s, 2} D_{s, 2}} \sim \frac{l_{1}}{s_{1} K_{s, 1} D_{s, 1}}
$$

Next we may consider water/salt selectivity. For synthetic membranes with aquaporin it becomes sensitive to the geometry of the membrane:

$$
\alpha_{w / s}=\frac{P_{w}}{P_{s}}=\frac{D_{w}}{K_{s, 1} D_{s, 1}} \frac{l_{1}}{l_{2}} \frac{s_{2}}{s_{1}}
$$

When salt and water permeability are limited by different layers, increase of aquaporin content on porous support, leading to increase of $s_{1}$, should only decrease water/salt selectivity. On contrary, in suspensions of biological membranes, where unstirred layers do not play significant role, selectivity of membranes with aquaporin is determined only by membranes, and is described by traditional equation:

$$
\alpha_{\text {aquaporin }}=K_{w, 1} D_{w, 1} / K_{s, 1} D_{s, 1}
$$

Now we can predict what relative content $s_{1} / s_{2}$ of aquaporin aqueous channels is necessary for the artificial membrane to have selectivity $\alpha_{\mathrm{w} / \mathrm{s}}$ :

$$
\frac{s_{1}}{s_{2}}=\frac{D_{w}}{K_{w, 1} D_{w, 1}} \frac{l_{1}}{l_{2}} \frac{\alpha_{\text {aquaporin }}}{\alpha_{w / s}}
$$

Aquaporin has very narrow channels ( $3 \AA$ in the narrow part), where water does not form mutual hydrogen bonds anymore. As the result, it is much more mobile than in a bulk volume (Agre 2004), and effective $D_{w, 1}$ in aquaporin channel is $\sim 30$ times larger than that in the solution. $K_{w, 1}$ has to be $\sim 1$ and $l_{1} / l_{2} \sim 5 \times 10^{-4}$. Assuming that $D_{w} / \mathrm{K}_{w, 1} D_{w, 1}=1 / 30$, to have selectivity of a membrane with porous support comparable to that of biological membrane with aquaporin, one has to have very low content of the channels $s_{1} / s_{2} \sim 1.7 \times 10^{-5}$. Even if diffusion coefficient in aquaporin is similar to that in bulk water, we still have $s_{1} / s_{2} \sim 5 \times 10^{-4}$, which should lead to low water permeability per unit of membrane area, making the whole process without external pressure not attractive for practice. Situation may be different in pressure-driven processes when diffusion through the porous support is not a rate-limiting factor anymore.

It is also possible to estimate the ratio $s_{1} / s_{2}$, which is necessary to have reported selectivity of artificial membranes $\alpha_{\mathrm{w} / \mathrm{s}}$ $=7500$ (Wang et al. 2012a). Typical permeability of oocytes with aquaporin for water, $\mathrm{H}^{+}$, and $\mathrm{K}^{+}$is $1.5 \times 10^{-4} \mathrm{~m} / \mathrm{s}, 10^{-6}$ $\mathrm{m} / \mathrm{s}$ and $1.5 \times 10^{-11} \mathrm{~m} / \mathrm{s}$, respectively, and it is practically zero for $\mathrm{Na}^{+}$(Wu et al. 2009). It means that water $/ \mathrm{K}^{+}$selectivity for aquaporin is $\sim 10^{7}$, and it is even better for water $/ \mathrm{Na}^{+}$. We assume again that $D_{w} / \mathrm{K}_{w, 1} D_{w, 1}=1 / 30$. If we use water $/ \mathrm{K}^{+}$selectivity for aquaporin $\left(10^{7}\right)$ instead of water/salt selectivity, Eq. 8 gives $s_{1} / s_{2} \sim 2.2 \times 10^{-2}$. This ratio is similar to the ratio of the surface area of a single aqueous channel $\left(\sim 10 \AA^{2}\right)$ and the surface taken by the channel-forming protein molecule $\left(\sim 400 \AA^{2}\right)$. For $\mathrm{Na}^{+}$the ratio $s_{1} / s_{2}$ should be much larger, but aquaporin can not cover more than $100 \%$ of the synthetic membrane. Even larger content would be necessary, if $D_{w} / \mathrm{K}_{w, 1} D_{w, 1}=1$, i.e. reported water $/ \mathrm{NaCl}$ selectivity seems physically impossible.

The raw data and equations to calculate major parameters were not given in the paper and instead of Arrhenius equation to calculate activation energy the authors gave equation similar to our Eq. 2. It states that water flux is proportional to the osmolarity difference and does not have activation energy at all.

The authors did not see the fundamental flaws in the results, but soon published Corrigendum, which stated that "The authors have found mistakes in Table 1 and related text due to an inappropriate calibration curve and unit conversion". They also wrote that the Eq. S3 and caption for Fig. 2 were wrong (Wang et al. 2012 b). After modification Table 1 had the same water flux but 20 times lower salt flux. 
Surprisingly, recalculated water to salt selectivity increased by a factor of two, and salt rejection decreased by less than $10 \%$ in the same Table 1.

It seems that the major problem with the way selectivity and salt rejection were calculated is that the authors used well-known equations for driven by pressure difference reverse osmosis. These equations do not include time. They are based on salt concentrations in the feed and the draw solution, respectively, when some volume went through the membrane and were used later in another publication from the same group (Wang et al. 2013a).

This time the process was driven by both external pressure ( 1 bar in this case) and osmotic pressure differences through the membrane. Hydrostatic pressure difference was negligible in these experiments. The transmembrane water flux through what they call "further improved" aquaporin Z-based membrane was only $17.6 \mathrm{l} / \mathrm{m}^{2} / \mathrm{h}$, and salt rejection was only $92 \%$ using $0.8 \mathrm{M}$ sucrose as the draw solution. When total of all driving factors is zero, water transport should evidently stop. Nevertheless, extrapolation of water flux as a function of solute concentration in a draw solution (Fig. 5 in Wang et al. 2013a) shows that without osmotic and external pressure differences the flux still was $\sim 10 \mathrm{l} / \mathrm{m}^{2} / \mathrm{h}$.

\section{Kinetics and thermodynamics}

One of the most important characteristics of any membrane transport is transmembrane flux, which is determined by total of all possible driving factors. In forward osmosis the major driving factor is osmotic pressure. In the presence of applied mechanical pressure the process is called pressureretarded osmosis. Relative role of these factors depends on the pore size, and for microfiltration and ultrafiltration usually the pressure-driven flux is dominant. Relation of pressure-driven and diffusion fluxes for different pore sizes was described previously (Kocherginsky 2010a).

When the transmembrane pressure difference is not applied, the flux has to be determined by diffusion. These are the conditions used in publication in Small. Initially the forward osmosis process may be practically irreversible for salt transport, but it is not for water. It is necessary to study transport kinetics (concentration and volume or weight changes in time). After corrections related to the volume and density changes it is possible to calculate permeability for water and salt, and, finally, for the steady state to calculate the selectivity as the ratio of permeabilities (Jin et al. 2011). This approach is good only if two fluxes do not influence each other, which is not necessary true for simultaneous transport of water and salt in forward osmosis. If one uses the ratio of fluxes where concentration changes with time, together with equations for salt rejection and permeability for reverse osmosis (as it was done in Eq. 3 and 4, Wang et al. 2013a), these parameters and selectivity will change with time, which does not make sense.

Forward osmosis is an emerging technology. It is important to have not only reproducible and correct experimental results, but also correct terminology and theoretical description for the flux, permeability and selectivity. In van't Hoff's experiments hydrostatic pressure difference through the membrane finally was balanced by osmotic pressure difference. This state when water transport stops is by definition an equilibrium state. In a later publication Professor Chung considers the steady state when the vesicles on porous support are not broken but water still moves through them from a feed to a draw solution, driven by osmotic pressure difference (Wang et al. 2013b). He gives his own definition of equilibrium: "The water flow through the vesicles at this stage is defined as the equilibrium water flux". Mathematical modeling conducted in this paper again did not consider effects of additional unstirred layers, but it was claimed that it is theoretically possible to achieve "equilibrium" water flux $150 \mathrm{l} / \mathrm{m}^{2} / \mathrm{h}$ using $180 \mathrm{~nm}$ vesicles with water permeability $6 \times 10^{-4} \mathrm{~m} / \mathrm{s}$.

In another Professor Chung's paper one can read that "according to the $2^{\text {nd }}$ law of thermodynamics, chemical potential tends to equilibrate in an isolated system". And further: solvents in forward osmosis move across the membrane "in order to equilibrate their overall chemical potential" (Ge et al. 2013). Different formulations of the $2^{\text {nd }}$ law may be found in textbooks. The older formulations are based on heat engines and more recent - on entropy, but there is no formulation based on Gibbs chemical potentials. The reason is clear. In traditional facilitated transmembrane transport ions may be concentrated, driven by $\mathrm{pH}$ gradient. In this case in equilibrium electrochemical (not chemical) potential difference for one ion is balanced by a similar difference for another ion, but even the electrochemical potentials of the same ion are not equal on different sides of the membrane. Similar factors are valid for any multicomponent and especially coupled transport, including that of water and ions in forward osmosis where solutions are not ideal.

To describe transmembrane transport of each component complicated by pressure and temperature difference instead of chemical or electrochemical potentials one could use more general physico-chemical potentials of different components (Kocherginsky 2010a, 2010b). In this case the relation of permeability in diffusion, electrophoresis, osmotic and external pressure-driven processes may be expressed as a function of the same suggested by Einstein's mobility. Kinetics and nonequilibrium thermodynamics of multicomponent transport, driven by many factors simultaneously, should be based on analyses of the gradients of physico-chemical potentials, their mutual influence and possible stoichiometry relations (Kocherginsky 2010c; Kocherginsky and Gruebele 2013). Both thermodynamics and kinetics of voltage-sensitive ion chan- 
nels and oxidative phosphorylation were discussed based on this approach previously (Kocherginsky 2009a, 2009b).

\section{Nitrocellulose-based biomimetic membranes}

We should also mention that aquaporin is not available on a market and probably will not be cheap. It is much easier and cheaper to make biomimetic membranes, which are based on simple nitrocellulose filters impregnated with esters of fatty acids (Kocherginsky et al. 1984, 1987, 1996; Kocherginsky and Lvovich 2010). The pore volume in this case is filled with oils, but still water enters the filters and covers internal surface of pores. Because of carboxylic groups on nitrocellulose these aqueous nanolayers (channels without proteins) are not only water-permeable, but also cation/anion selective. $\mathrm{NaCl}$ practically did not penetrate through the membrane, but its gradient changed transmembrane voltage. Water may be transported through the membrane by osmotic pressure difference, which may be seen both using tritium-labeled water and simply based on changes of volumes on both sides of the membrane. It was possible to imitate many barrier and other physico-chemical properties of biomembranes, including phase transitions and interactions with some drugs, which means that even without proteins nitrocellulose-based biomimetic membrane may be considered as a simplest biomimetic prototype of a membrane with selective aqueous channels for forward osmosis.

\section{Final comment}

When this manuscript was practically ready, Professor Chung published one more paper, where permeability and selectivity of a membrane with imprinted aquaporinbased vesicles was reported (Xie et al. 2013). The authors claim that this further improved and mechanically stable membrane may be used in low energy water filtration, but the energy characteristics of the process in this paper were not given. Water flux with $0.3 \mathrm{M}$ sucrose as the draw solution was near $5.6 \mathrm{l} / \mathrm{m}^{2} / \mathrm{h}$. Though $\mathrm{NaCl}$ rejection was only $50.7 \%$, water/salt selectivity was as high as $3.35 \times 10^{4}$. Even without aquaporin, when salt rejection by vesicle-imprinted membranes was zero, water/salt selectivity (the way it was calculated) was as high as 10000 (Table 1 and Eq. 4 in Xie et al. 2013). The reasons for this contradiction were not discussed, and the publication in the journal Small was not mentioned.

Acknowledgement. All experimental work on aquaporin-based membranes was supported by Singapore National Research Foundation under its Competitive Research Program, the project "Advanced FO membranes and membrane systems for water treatment, water reuse and sea water desalination" (grants R-279-000-336-281 and R-279-000-293-272), and EWI project 0804-IRIS-01.

\section{References}

Agre P. (2004): Aquaporin water channels (Nobel lecture). Angewandte Chemie-International Edition 43, 4278-4290 http://dx.doi.org/10.1002/anie.200460804

Baker R. W. (2004): Membrane Technology and Applications. J. Wiley \& Sons, Chichester, England http://dx.doi.org/10.1002/0470020393

Ge Q., Ling M., Chung T.-S. (2013): Draw solutions for forward osmosis processes: Developments, challenges, and prospects for the future. J. Memb. Sci. 442, 225-237 http://dx.doi.org/10.1016/j.memsci.2013.03.046

Guofei S., Hu Z., Yi L., Jeyaseelan K., Armugam A., Chung T.-S. (2012): A novel method of AquaporinZ incorporation via binary-lipid Langmuir monolayers. Colloids and Surfaces B: Biointerfaces 89, 283-288 http://dx.doi.org/10.1016/j.colsurfb.2011.09.004

Jin X., Tang, C. Y., Gu Y., She Q., Qi S. (2011): Boric acid permeation in forward osmosis membrane processes: modeling, experiments, and implications. Environ. Sci. Tech. 45, 2323-2330 http://dx.doi.org/10.1021/es103771a

Kocherginsky N. M., Bromberg L. E., Osak I. S., Moshkovsky Y. S., Popkov Y. M., Skuratnik Y. B. (1984): Water transport in biological membranes modeled using impregnated filters. Biological Membranes (Biologicheskie Membrany) 1, 954-963 (in Russian), 1734-1743 (English translation 1985)

Kocherginsky N. M., Osak I. S., Bromberg L. E., Karyagin V. A., Leskin G. S, Moshkovsky Yu. S. (1987): The modeling of biological membrane properties by means of filters impregnated with lipid-like substances. J. Memb. Sci. 30, 39-46 http://dx.doi.org/10.1016/S0376-7388(00)83339-6

Kocherginsky N. M., Liu K. J., Swartz H. M. (1996): Thermoinduced phase transitions and regulation of permeability of biomimetic membranes. In: Biofunctional Membranes (Ed. D. A. Butterfield), pp.163-172, Plenum Press, New York http://dx.doi.org/10.1007/978-1-4757-2521-6_11

Kocherginsky N. (2009a): Voltage-sensitive ion channels, acidic lipids and Hodgkin-Huxley equations: New ideas 55 years later. J. Memb. Sci. 328, 58-74 http://dx.doi.org/10.1016/j.memsci.2008.10.024

Kocherginsky N. (2009b): Acidic lipids, H+-ATPases, and mechanism of oxidative phosphorylation. Physico-chemical ideas 30 years after P. Mitchell's Nobel Prize award. Prog. Biophys. Mol. Biol. 99, 20-41 http://dx.doi.org/10.1016/j.pbiomolbio.2008.10.013

Kocherginsky N. M. (2010a): Mass transport and membrane separations: universal description in terms of physicochemical potential and Einstein's mobility. Chem. Eng. Sci. 65, 1474-1489 http://dx.doi.org/10.1016/j.ces.2009.10.024

Kocherginsky N. M. (2010b): Temperature-driven mass, charge and heat transfer in terms of physicochemical potential and Einstein's mobility. Chem. Eng. Sci. 65, 4154-4159 http://dx.doi.org/10.1016/j.ces.2010.04.035

Kocherginsky N. M. (2010c): Semi-phenomenological thermodynamic description of chemical kinetics and mass transport. Journal of Non-Equilibrium Thermodynamics 35, 97-124 http://dx.doi.org/10.1515/jnetdy.2010.007 
Kocherginsky N. M., Lvovich V. F. (2010): Biomimetic membranes with nano channels but without proteins. Impedance of impregnated cellulose esters filters. Langmuir 26, 18209-18218 http://dx.doi.org/10.1021/la102345t

Kocherginsky N., Gruebele M. (2013): A thermodynamic derivation of the reciprocal relations. J. Chem. Phys. 138, 124502 http://dx.doi.org/10.1063/1.4793258

Kumar M., Grzelakowski M., Zilles J., Clark M., Meier W. (2007): Highly permeable polymeric membranes based on the incorporation of the functional water channel protein Aquaporin Z. Proc. Nat. Acad. Sci. USA 104, 20719-20724 http://dx.doi.org/10.1073/pnas.0708762104

Li X., Wang R., Tang C., Vararattanavech A., Zhao Y., Torres J., Fane T. (2012): Preparation of supported lipid membranes for aquaporin $\mathrm{Z}$ incorporation. Colloids and Surfaces B - Biointerfaces 94, 333-340 http://dx.doi.org/10.1016/j.colsurfb.2012.02.013

Loeb S., Sourirajan S. (1963): Sea water demineralization by means of an osmotic membrane. In: Saline Water Conversion II (Ed. R.F. Gould), Advances in Chemistry, Series number 38, pp. 117-132, American Chemical Society, Washington, DC

Phuoc H. H. Duong, Chung T.-S., Jeyaseelan K., Armugam A., Chen Z., Yang J., Hong M. (2012): Planar biomimetic aquaporin-incorporated triblock copolymer membranes on porous alumina supports for nanofiltration. J. Memb. Sci. 409, 34-43

van't Hoff J. H. (1995): The role of osmotic pressure in the analogy between solutions and gases. J. Memb. Sci. 100, 39-44 http://dx.doi.org/10.1016/0376-7388(94)00232-N

Wang H., Chung T.-S., Tong Y. W., Jeyaseelam K., Armugam A., Chen Z., Hong M., Meier W. (2012a): Highly permeable and selective pore-spanning biomimetic membrane embedded with aquaporin Z. Small 8, 1185-1190 http://dx.doi.org/10.1002/smll.201102120
Wang H., Chung T.-S., Tong Y. W., Jeyaseelam K., Armugam A., Chen Z., Hong M., Meier W. (2012b): Corrigendum. Highly permeable and selective pore-spanning biomimetic membrane embedded with aquaporin Z. Small 8, 1969 http://dx.doi.org/10.1002/smll.201201148

Wang H., Chung T.-S., Tong Y. W., Jeyaseelan K., Armugam A., Phuoc Duong H. H., Fu F., Seah H., Yang J., Hong M. (2013a): Mechanically robust and highly permeable Aquaporin $\mathrm{Z}$ biomimetic membranes. J. Memb. Sci. 434, 130-136 http://dx.doi.org/10.1016/j.memsci.2013.01.031

Wang H., Chung T.-S., Tong Y. W. (2013b): Study on water transport through a mechanically robust Aquaporin $\mathrm{Z}$ biomimetic membrane. J. Memb. Sci. 445, 47-52

http://dx.doi.org/10.1016/j.memsci.2013.05.057

Wu B., Steinbronn C., Alsterfjord M., Zeuthen T., Beitz E. (2009): Concerted action of two cation filters in the aquaporin water channel. EMBO J. 28, 2188-2194 http://dx.doi.org/10.1038/emboj.2009.182

Xie W., He F., Wang B., Chung T.-S., Kandiah J., Armugamb A., Tong Y. W. (2013): An aquaporin-based vesicle-embedded polymeric membrane for low energy water filtration. J. Mater. Chem. A 1, 7592-76700 http://dx.doi.org/10.1039/c3ta10731k

Zhao Y., Qiu C., Li X., Vararattanavech A., Shen W., Torres J., Claus H.-N., Wanga R., Hua X., Fane A. G., Tang C. Y. (2012): Synthesis of robust and high-performance aquaporin-based biomimetic membranes by interfacial polymerization-membrane preparation and RO performance characterization. J. Memb. Sci. 423, 422-428 http://dx.doi.org/10.1016/j.memsci.2012.08.039

Zhong P. S., Chung T.-S., Jeyaseelan K., Armugam A. (2012): Aquaporin-embedded biomimetic membranes for nanofiltration. J. Memb. Sci. 407, 27-33 http://dx.doi.org/10.1016/j.memsci.2012.03.033 